\title{
Report on the US-Japan Workshop - Plasma Fueling and Active Particle Control
}

Keith I. Thomassen

March 1, 2000

U.S. Department of Energy

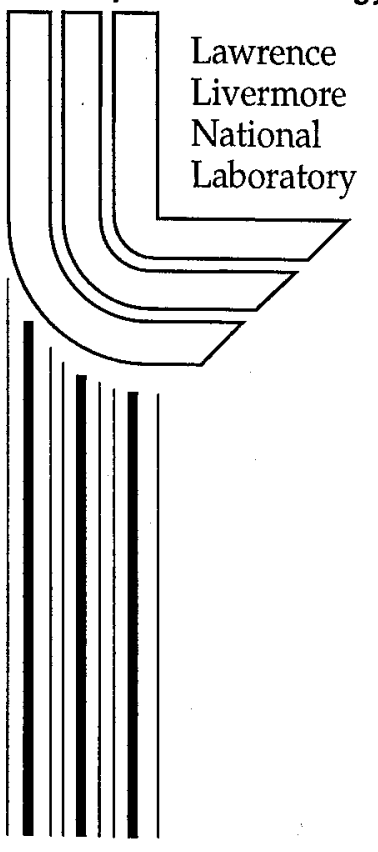




\section{DISCLAIMER}

This document was prepared as an account of work sponsored by an agency of the United States Government. Neither the United States Government nor the University of California nor any of their employees, makes any warranty, express or implied, or assumes any legal liability or responsibility for the accuracy, completeness, or usefulness of any information, apparatus, product, or process disclosed, or represents that its use would not infringe privately owned rights. Reference herein to any specific commercial product, process, or service by trade name, trademark, manufacturer, or otherwise, does not necessarily constitute or imply its endorsement, recommendation, or favoring by the United States Government or the University of California. The views and opinions of authors expressed herein do not necessarily state or reflect those of the United States Government or the University of California, and shall not be used for advertising or product endorsement purposes.

Work performed under the auspices of the U.S. Department of Energy by the University of California Lawrence Livermore National Laboratory under Contract W-7405-Eng-48.

This report has been reproduced

directly from the best available copy.

Available to DOE and DOE contractors from the

Office of Scientific and Technical Information

P.O. Box 62, Oak Ridge, TN 37831

Prices available from (423) 576-8401

http://apollo.osti.gov/bridge/

Available to the public from the

National Technical Information Service

U.S. Department of Commerce

5285 Port Royal Rd.,

Springfield, VA 22161

http://www.ntis.gov/

OR

Lawrence Livermore National Laboratory

Technical Information Department's Digital Library

http://www.llnl.gov/tid/Library.html 


\title{
Report on the US-Japan Workshop
}

\author{
Plasma Fueling \\ and Active Particle Control \\ March 1-3, 2000, LLNL
}

K. Thomassen, Editor

Contributors:
L. Baylor
F. Bombarda
E.B. Hooper
D. Hwang
N. Fukumoto
K. Kizu
H. McLean
T. Mizuuchi
T. Morisaki
O. Motojima
Y. Nakashima
T. Petrie
G. Porter
H. Suzuki
T. Rognlien
H. Yamada
M. Wade
Z. Yoshida 
The third in a series of these workshops was held, at LLNL as was the first one. In the first workshop 2 years ago, emphasis was on particle fueling and technologies. Last year at Toki, this was augmented by science results involving fueling experiments. This year, much more attention was placed on science, and the subject matter was broadened to include active particle control and the results of experiments attempting to do so.

Many interesting papers were presented, and their key results are summarized by the presenters listed below (only the presenter, not the multiple authors, are listed). This summaries follow the agenda for the workshop.

Results of the performance in LHD with pellet fueling and higher power heating systems were presented, along with work on a local island divertor and a membrane pump. The new Heliotron $J$ is in operation, a follow-on to Heliotron $E$ at Kyoto. Results of inside launch pellets in JT-60U and DIII-D were given, and particle control in the advanced divertor on DIII-D was described. Modeling results on core fueling from divertor recycling in DIII-D were given, fueling for Ignitor was described, and fueling studies on GAMMA 10 were presented. A paper was given on particle control for liquid surfaces, and the activities at ORNL on fueling were summarized. CT injection experiments in the Davis Tokamak, and injection of pellets across an external field at Himeji were described. Finally, particle control in SSPX was discussed, and a very nice set of theory papers on self-organization from Univ. of Tokyo was given. They describe a modification to the theory when shearflows exist, and a two-fluid description is required.

\section{DEVELOPMENT AND FIRST RESULTS OF THE CENTRIFUGAL PELLET INJECTOR FOR JT-60U (K. Kizu)}

In ITER operation, high confinement and high density plasmas must be sustained. However, as observed in JT-60U, when increasing the electron density by gas puffing, the confinement is drastically degraded. Therefore, core fueling by repetitive pellet injection is expected to improve the confinement property. Thus, a centrifugal pellet injector was developed in JT-60U. A guide tube for magnetic high field side (HFS) injection was also developed. To investigate the performance of the injector, the first pellet injection experiments from magnetic low field side (LFS) and HFS were conducted using $\mathrm{OH}$ - plasmas.

The developed centrifugal pellet injector can eject 30-40 pellets with frequency $10 \mathrm{~Hz}$, speed $1.0 \mathrm{~km} / \mathrm{s}$, and pellet size $1.9 \mathrm{~mm}$ cubic $(<700 \mathrm{~m} / \mathrm{s})$. Key techniques for the development were acceleration components with a gas removing mechanism and a tapered pellet guide tube (funnel) with appropriate pellet impact angle. By using such acceleration components, the horizontal angular distribution of ejected pellets became narrow and an injection efficiency of over $80 \%$ was achieved. This indicates that the sublimated gas from pellets make the pellet motion irregular.

First pellet injection experiments were performed from the LFS using an $\mathrm{OH}$ plasma. Pellets $300-700 \mathrm{~m} / \mathrm{s}$ were successfully injected without destruction because of the optimized funnel. The central fuelling and enhanced fuelling rate were observed. Thus, D-alpha intensity around the divertor region was smaller than for 
the gas puffing case, indicating that low recycling was maintained during pellet injection.

The guide tube for HFS injection has a total length of about $15 \mathrm{~m}$, with $5 \mathrm{~mm}$ inner diameter. The minimum curvature is $600 \mathrm{~mm}$. Results of HFS injection with pellet speeds over $320 \mathrm{~m} / \mathrm{s}$ implied pellet destruction through the guide tube. However, injection efficiency was improved by reducing the pellet speed to $220 \mathrm{~m} / \mathrm{s}$. Pellet mass was also increased up to about $68 \%$ of LFS injection.

\section{FUELING TECHNOLOGY DEVELOPMENTS AT ORNL (L. Baylor)}

Improved fueling efficiency from inside launched pellets on ASDEX-Upgrade and DIII-D has inspired many other devices to investigate alternative pellet injection locations using curved guide tubes to transport pellets to locations other than the outside midplane. Tests of pellet survival in curved guide tubes have been undertaken at ORNL to support the design of alternative injection locations. Pellet speeds in excess of $400-500 \mathrm{~m} / \mathrm{s}$ appear to be problematic for intact pellet transmission due to cumulative stress buildup from multiple collisions with the guide tube surface. Special techniques are often required to achieve slow enough pellets to survive guide tube designs. Pellet feed systems using extruders have been developed that can supply up to 700 pellets from single extrusions. Multiple extruders have been shown to be capable of providing hydrogenic ice for steady state pellet fueling. Technology related to pellet fueling is under development for disruption mitigation of tokamak plasmas. A massive gas puff device has been used on DIII-D with success and liquid jet technology is under development. Jets with long lengths relative to the diameter are needed with large Reynolds and Weber numbers that will push beyond the state of the art in liquid jet technology.

\section{FUELING STUDIES IN THE GAMMA 10 TANDEM MIRROR (Y. Nakashima)}

In order to increase density in the GAMMA 10 tandem mirror, pellet injection experiments have been started. A bench test of the pellet injector showed that the system has a capability to produce sub-millimeter hydrogen ice-pellets to launch through aTeflon guide tube into the GAMMA 10 plasma. By injecting the submillimeter pellets into the central-cell, a significant increase of the line density in the central-cell plasma was observed together with valuable signals of $H_{\alpha}$ emission, soft X-ray and end loss ion flux.

Potential confinement of pellet-fuelled plasma was succesfully demonstrated and the plasma parameter space was extended using this fueling. As a future task, an investigation of radial transport processes of the pellet-fuelled particles is needed. An experiment of pellet injection into the central ECRH mode plasmas is planned in the near future to improve the energy confinement during pellet fueling.

\section{FUELING REQUIREMENTS FOR IGNITOR (F. Bombarda)}

Ignitor is a compact, high field machine designed to reach ignition in ohmically heated plasmas, at high density $\left(n_{0} \sim 10^{21} \mathrm{~m}^{-3}\right)$ and relatively low temperatures $\left(T_{e} \sim T_{i} \sim 11 \mathrm{keV}\right)$. It will operate well below the density and beta limits. Ignition is most readily achieved when adequately peaked density profiles are established, as good confinement properties are usually associated with them. 
Plasma regimes with Internal Transport Barriers should also be relatively easy to achieve, with the help of ICRF heating.

Time dependent simulations of Ignitor plasmas at the reference (11 MA, $13 \mathrm{~T}$, with the JETTO code) or at the maximum (12 A, $13 \mathrm{~T}$, BALDUR code) parameters indicate that particle fluxes at the plasma surface will be of the order of $4 \times 10^{20}$ to 8 $x 10^{21}$ particles $/ \mathrm{m}^{2} \mathrm{~s}$ ). These values are not too far from what is observed, for example, on Alcator C-Mod. Nevertheless, it may prove difficult to fuel the discharge with gas puffing alone. Therefore, a pellet injector has always been considered an integral part of the machine design to perform a number of tasks, such as; (fast) core fueling, density profile control, controlled tritium injection, active burn control, promote the formation of ITB's, and serve diagnostic purposes with doped pellets.

Given the strongly dynamical condition of the Ignitor plasmas, the pellet injector will have to be a highly flexible device, allowing for example pellets of different sizes, controllable speed up to $2.5-3 \mathrm{~km} / \mathrm{sec}$, simultaneous injections, repetition rates up to several $\mathrm{Hz}$, use of different gas, etc. These characteristics represent a combination of features already achieved, separately, on existing injectors.

The recent results from HFS injection are of interest, of course, as they may help relax some of the most demanding requirements. However, the limited vertical access and the compact shape of the machine presumably will not allow vertical pellet launch at less than a couple of centimeters to the inboard side of the magnetic axis.

\section{WALL PUMPING OF THE LHD AND MEMBRANE PUMP (H.Suzuki)}

Particle balance in a plasma vacuum vessel of the Large Helical Device (LHD) has been studied using a fast ionization gauge (FIG), which is operational in a high magnetic field. The plasma vacuum vessel, made of stainless steel, has a total volume of $210 \mathrm{~m}^{3}$ and total area of $730 \mathrm{~m}^{2}$. Two carbon plates with a total area of $3 \mathrm{~m}^{2}$ are installed on the vessel wall as NBI armor plates. These were the only carbon plates in the vacuum vessel during the second campaign of LHD. After the second campaign, helical divertor plates, with a total area of $30 \mathrm{~m}^{2}$, were installed.

The LHD experiments have been carried out using hydrogen and helium plasmas. Using gas puffing data and a pressure curve during a plasma shot, the particle balance was investigated. There were very few missing particles in measurements from helium shots during the second campaign. On the other hand, almost half of the input particles were missing in hydrogen shots, attributed to the wall pumping effect. High energy hydrogen atoms, which were produced by charge exchange interaction, were implanted into the stainless steel wall.

No saturation of the wall was observed during the second and third campaign of LHD. The total accumulation of hydrogen atoms in the stainless steel depends on lattice defects, so it is not easy to predict when the wall will be saturated. A more controllable pump is required. The superpermeable membrane pump is a new concept for wall pumping. Certain metals like $\mathrm{Nb}, \mathrm{V}, \mathrm{Ta}, \mathrm{Pd}$ show a special phenomenon, which is called "superpermeation" Hydrogen atoms can pass through a membrane of such metals with a permeation probability of nearly unity.

Only hydrogen atoms can pass through it --- hydrogen molecules or other atoms cannot. If a hydrogen atom source, such as a plasma, is put on one side of the superpermeable membrane, the hydrogen atoms pass through the membrane, 
and combine into hydrogen molecules. Consequently, hydrogen passes the membrane in only one direction, and this is the principle of the membrane pump. The membrane pump was operated in a test stand and it worked successfully. We are now planning to install a superpermeable membrane plate in LHD as a first step towards studying the effect of impurities such as carbon or iron on the pump.

\section{CHARACTERIZATION OF PLASMA CONFINEMENT RELATED TO FUELING CONDITIONS IN LHD (H.Yamada)}

Fueling by gas puffing is a primary concern in LHD, as has been discussed in this workshop series and in this study. Neutrals are ionized outside the last closed flux surface in large high-temperature plasmas, which leads to significant deterioration of fueling efficiency. Recent LHD experiments have convinced us of this issue. The fueling rate limits plasma confinement performance as well as the accessible density in a limited pulse length. Pellet injection has made a tremendous impact on extending the operational regime and performance limits in LHD. The dynamics that limit the pedestal pressure, which is an essential element of confinement improvement, are found in the period after pellet injection. So long as there exists room for pressure gradients in the edge region, the energy confinement time shows a large improvement. However, when the pressure of the pedestal reaches a certain level, there are bursts of $H_{\alpha}$ and magnetic fluctuations are excited, limiting the pressure increase. Particles are missing after a redistribution period immediately after the pellet injection, as also observed in tokamaks. High field side (HFS) injection experiments are ready and comparison with tokamak HFS results will be stimulating.

THE EFFECT OF DIVERTOR SHAPE AND PUMPING ON DENSITY LIMIT AND CONFINEMENT IN DIII-D (T.W. Petrie)

The presentation discussed factors which either hinder or facilitate high density plasma operation with good energy confinement during deuterium gas injection. Cases with and without active particle pumping were considered. We discussed the roles of "divertor" and "core" Marfes during gas puffing at high density and showed that their suppression (especially the latter) is essential for high density operation with good confinement. "Natural" suppression of the Marfes, in fact, is largely responsible for the observed higher densities achieved in double-null divertors than in comparable single-null divertors, which can form Marfes relatively easily. We also showed that, while high "triangularity" of a plasma discharge at low density has significantly higher energy confinement than low triangularity discharges also at low density, this advantage disappears at higher densities during gas puffing. Finally, we demonstrated that it is possible to achieve high density ( 1.4 times the Greenwald limit) and good H-mode confinement ( 2x ITER-89) under certain operating conditions in DIII-D. The prescription on how to accomplish this was discussed.

ACTIVE EDGE CONTROL BY LOCAL ISLAND DIVERTOR (T. Morisaki) 
The principles of the local island divertor (LID) and experimental results in the compact helical system (CHS) were presented, which is the first application to toroidal plasmas. In the experiment, it was clearly demonstrated that particles diffused out from the confinement region and are guided to the back side of the divertor head, following the separatrix of the superimposed $m / n=1 / 1$ island. In addition to the demonstration of some fundamental divertor functions, a modest improvement of energy confinement was observed, which could be attributed to the edge plasma control by LID.

For the large helical device (LHD), numerical simulations to study the charged and neutral particle behavior in the LID configuration have been performed simultaneously. For charged particles, a field line tracing code coupled with a random walk process, simulating the diffusion, gives the strike-point distribution on the divertor head. It was found that particles can identify and follow the island structure if the diffusion coefficient is less than $0.1 \mathrm{~m}^{2} / \mathrm{sec}$. So, LID works well as a divertor and the head is free from a leading edge problem, which would be a severe one in steady state operation. On the other hand, LID acts like a conventional limiter for diffusion coefficients larger than $0.1 \mathrm{~m}^{2} / \mathrm{sec}$. For neutral particles, a 2D Monte Carlo code (DEGAS) is utilized to calculate the pumping efficiency of LID, and it was shown that an efficiency up to $50 \%$ can be achieved for low recycling operation.

The present status of constructing a LID for LHD was presented.

\section{PARTICLE CONTROL WITH LIQUID WALLS AND DIVERTOR SURFACES (T.D. Rognlien)}

Using liquid walls and divertors are attractive for fusion power-plants for a number of reasons including the following: greatly reduced wall damage and activation, up to an order of magnitude increase in allowable neutron wall loading, and high heat-flux divertor capability. These improvements could reduce the cost of electric by $35 \%$. We study the important issue of the effectiveness of edge-plasma shielding of the core plasma from impurities generated by the evaporating wallvapor. A large tokamak is analyzed with the UEDGE 2-D edge-plasma transport code. A lithium liquid surfaces can pump hydrogen effectively, giving a lowrecycling edge plasma with low divertor density and high temperature. Other liquids, such as Flibe or LiSn (20/80) recycling hydrogen, yielding a high divertor density, lower temperature high-recycling divertor regime. For a given core-edge hydrogen density, the low-recycling edge plasma shields the core better from impurities compared to high-recycling because of the rapid parallel flow of impurities to the divertor region. However, the low-recycling regime will likely run at lower core-edge hydrogen density owing to the rapid particle loss, which will require strong particle fueling by pellets or some other mechanism. At the lower edge density, some of the advantage of the low-recycling regime giving better shielding is lost; more detailed studies of the lower density regime are being conducted.

EXPERIMENTAL STUDIES ON EDGE PLASMA CONTROL IN HELIOTRON J (T. Mizuuchi) 
A new advanced post-Heliotron $\mathrm{E}$ device, Heliotron $\mathrm{J}$, device, has started operations at the Institute of Advanced Energy in Kyoto University. Heliotron $\mathrm{J}$ $\left(\mathrm{L}=1, \mathrm{M}=4\right.$, with $\left.\mathrm{R}=1.2 \mathrm{~m},\left\langle\mathrm{a}_{\mathrm{p}}\right\rangle=0.15-0.2 \mathrm{~m}, \mathrm{~B}=1.5 \mathrm{~T}\right)$ is designed to realize a helical axis heliotron configuration, newly proposed based on the experience of previous heliotron experiments and recent theoretical understandings. The main purposes of Heliotron $\mathrm{J}$ experiment are;

- to comprehend the basic physics in the advanced toroidal plasma confinement as a fusion research facility in a university setting.

- to demonstrate the improved confinement based on the helical axis heliotron concept , and

- to get the design principles for the next step (POP) device based on this concept.

In this paper, experimental plans for particle control in Heliotron $\mathrm{J}$ (recycling control, fueling control and divertors) are discussed by comparing the experimental results with our previous device, Heliotron E. In Helotron J, "wall pumping (low recycling wall)" and deeper fueling are required for density (profile) control as in Heliotron E. To get "wall pumping" (low recycling wall), Ti-getterng is used. In the near future, boronization with di-borane will be applied. For deeper fueling, pellet and/or $\mathrm{NBI}$ injection is planned. Heliotron $\mathrm{J}$ has several local divertor configurations, and a design study of the island divertor structure is just started.

\section{IMPROVED PELLET FUELING IN DIII-D}

L. Baylor

Pellet injection has been successfully employed on DIII-D from different injection locations to optimize the mass deposition for density profile control and internal transport barrier formation. Pellets injected from inside the magnetic axis from the inner wall or vertical injection port lead to stronger central mass deposition than pellets injected from the low field side (LFS) and thus yield deeper, more efficient, fueling. An outward major radius drift of the pellet mass during the toroidal symmetrization process is suggested by the resulting deposition profiles. Transport barriers have been formed deep in the plasma core with central mass deposition from high field side (HFS) injected pellets and in the edge with shallow penetrating pellets that trigger L-mode to $\mathrm{H}$-mode transitions. HFS pellet injection is a unique enabling technology for providing new physics understanding on DIII-D.

\section{ACHIEVEMENTS AND PROSPECTS OF LHD PROJECT O. Motojima}

In the third campaign (July 13th - December 14th in 1999), 4.2 MW of NBI, 1.4 MW of ICRH and $0.9 \mathrm{MW}$ ofECRH were used to heat plasmas in LHD. Carbon tiles were installed as divertor plates, resulting in a significant reduction in metal impurity concentration $(\mathrm{Fe})$. The maximum field used in the experiment is $2.9 \mathrm{~T}$. Upgrading the key hardware systems led to (i) higher $T_{e}$, reaching $T_{e}(0)=4.4 \mathrm{keV}$ at $n_{e}=5.3 \mathrm{x}$ $10^{18} \mathrm{~m}^{-3}$ and $P_{a b s}=1.8 \mathrm{MW}$, (ii) higher confinement time, $\tau_{E}=0.3 \mathrm{~s}$ when $T_{e}(0)=$ $1.1 \mathrm{keV}$ at $\mathrm{n}_{\mathrm{e}}=6.5 \times 10^{19} \mathrm{~m}^{-3}$ and $\mathrm{P}_{\mathrm{abs}}=2.0 \mathrm{MW}$, (iii) higher stored energy $W_{\mathrm{p}}$ dia $=$ $880 \mathrm{KJ}$, (iv) higher average $\left\langle\beta_{\mathrm{dia}}\right\rangle=2.4 \%($ at $\mathrm{B}=1.3 \mathrm{~T}$ ) than those in the second experimental campaign in 1998. The temperature pedestal enhances the 
confinement significantly ( $50 \%$ higher than the ISS95 scaling). The ICRH heating was found to be as efficient as NBI heating. Long pulse discharges ( $80 \mathrm{sec} \mathrm{NBI}$ shot, $68 \mathrm{sec}$ ICRH shot) have been achieved without any technical difficulty. The rapid progress of the plasma parameters is realized by the disruption free property of the LHD magnetic configuration and technical advantages of superconducting device. The LHD experiment is now exploring a new regime for toroidal magnetic confinement with currentless plasma, which is the least free energy configuration. It will provide a valuable database with a wide dynamic range for plasma physics and controlled fusion research.

\section{ACTIVE PARTICLE CONTROL WITH THE DIII-D ADVANCED TOKAMAK DIVERTOR (M. Wade)}

Density control has been identified as a "critical path" issue in lengthening the duration of recent high performance $\left(\beta_{\mathrm{N}} \approx 3.4, \mathrm{H}_{89} \approx 2.6\right)$ regimes obtained on DIII-D. Significant off-axis ECCD will be required to maintain an optimum current density profile is these plasmas. To control density, a new divertor hardware upgrade, including a private flux dome and cryopump in the upper-inner divertor of DIII-D, has just been completed.

This new hardware produces a closed divertor geometry (known as RDP2000) that can be coupled effectively to high $\delta$, high $\kappa$ plasma configurations. Studies using this new divertor configuration have shown that good density control $\left(n_{e} \sim 0.3 n_{G W}\right.$ and central $\left.Z_{\text {eff }} \sim 1.5\right)$ can reliably be achieved in ELMing $\mathrm{H}$-mode discharges with good normalized confinement $\left(\mathrm{H}_{89}=2.5\right)$.

Measurements show that the core ionization source is reduced by a factor of 3 in these discharges, relative to the open divertor geometry of the lower divertor in DIII-D. Also, induced SOL flow (i.e., puff and pump) studies have shown that the core impurity content of medium- $Z$ seeded impurities, such as neon and argon, can be reduced dramatically by strong $D_{2}$ fueling in the midplane region. Studies are just commencing on using this hardware for density control in high performance plasmas. Such density control is required to achieve adequate electron cyclotron current drive $(E C C D)$ in these plasmas.

\section{SELF-ORGANIZATION OF SHEAR-FLOW BOUNDARY LAYER IN H-MODE TOKAMAK (Z. Yoshida)}

When an appreciable strength of shear flow is given to a plasma, the set of self-organized states becomes far richer than the conventional prediction. It is the two-fluid effect that brings about an essential coupling among the flow, magnetic field, electric field and the pressure, resulting in a "singular perturbation" to the MHD system. To invoke this effect (which naturally shrinks in normal conditions), one must supply a drive to sustain a strong shear flow. It is equivalent to giving an internal electric field (non-neutralizing) or applying a steep gradient in pressure, since these fields are self-consistently coupled.

Mahajan and Yoshida have shown that the hydrodynamic pressure of a shear flow yields a diamagnetic state that is suitable for confining a high-beta plasma. The $\mathrm{H}$-mode boundary layer is an example of such structures, which are spontaneously generated by the core plasma pressure. The Beltrami/Bernoulli conditions describe the relaxed state of a general vortex dynamics system; the 
Beltrami condition demands that the vorticity parallels the flow, and the (generalized) Bernoulli condition implies that the energy density takes a flat distribution. In two-fluid MHD plasmas, these conditions read as a simultaneous system of equations both for electrons and ions. By the Beltrami conditions, electrons flow in the direction of the magnetic field $B$, while the ion flow (v) parallels $\mathbf{B}+\nabla \times \mathbf{v}$ (the second term represents the Coriolis force). A linear combination of two force-free fields (Taylor relaxed states) solves these simultaneous Beltrami conditions, but the combination is no longer force free. The pressure is given by the Bernoulli conditions, $p_{e}-n e \phi=$ constant and $p_{1}+n e \phi+M n \frac{v^{2}}{2}=$ constant $\left(p_{e}\right.$ and $p_{i}$ are the electron and ion pressures, $\mathrm{n}$ is the number density, $\phi$ is the electric potential, $M$ is the ion mass). Adding both equations, we obtain $\beta+V^{2}=$ constant (velocity $V$ in Alfven units).

Active control of shear flow will significantly extend the realizability of such self-organized states. We have proposed a new concept of high-beta confinement applying a non-neutralized plasma. We are at the stage of exploring an innovative method to inject electrons into a toroidal system. At the first series of experiments, pure electron plasmas of density $\sim 10^{13} \mathrm{~m}^{-3}$ and potential $\sim 500 \mathrm{~V}$ have been produced by injecting beam electrons into the Proto-RT device. Secondly, beam electrons have been injected into a weakly ionized plasma produced radiofrequency discharge. Inside the plasma, an excess electron flux with a decrease of plasma potential is observed at some measurement points, showing that the plasma is locally non-neutralized by the excess of electrons.

\section{MODELING OF CORE FUELING FROM DIVERTOR RECYCLING IN DIII-D (G.D. Porter)}

This paper described the core refueling resulting from neutral recycling in the divertor region of DIII-D. The refueling rates were determined both from analysis of experimental data, and from detailed modeling with the 2D fluid code UEDGE. The radial particle flux is determined experimentally by analysis of the radial density and temperature gradients which are measured by Thomson scattering. It was shown that the radial particle flux at the top of the $\mathrm{H}$-mode pedestal (the $96 \%$ poloidal flux surface) was comparable to the particle input from neutral beam heating. In contrast, the particle flux across the separatrix is typically four or five times the flux expected from neutral beam fueling. The separatrix particle flux increases when gas is introduced into the main chamber. In fact, the increase in the separatrix particle flux exceeds the input rate of the cold gas, suggesting amplification by recycling in the divertor. Only 2 to $3 \%$ of the neutral gas introduced in the main chamber penetrates the closed flux surfaces sufficiently to fuel the core plasma. The remainder fuels only the edge plasma, and recycles in the divertor. Modeling indicates the neutrals recycling from the divertor flow around the periphery of the scrape-off layer plasma to regions where the SOL is thin, then penetrates to the closed surfaces. Only about $1 \%$ of the neutral flux off the divertors penetrates to the closed lines.

RECENT EFFICIENCY IMPROVEMENTS AND EXPERIMENTAL RESULTS ON THE REPETITIVE COMPACT TOROID ACCELERATOR AT UC DAVIS

D. Q. Hwang 
Due to the passive formation of the SCT in the CTIX accelerator, gas feeding in the electrode region is critical to both the overall gas efficiency of the accelerator and the final parameters of the SCT. We had been able to increase the gas efficiency by a factor of 100 and the potential repetition rate by a factor of 1000 through the improvement of the gas feeding system and the modification of the accelerator electrode. At present, a repetitive rate of $1 \mathrm{kHz}$ in burst-mode is possible, the limitation arising from the gas handling capability of the DDT vacuum system and the energy storage capacity of the power system.

Another ongoing effort is modeling of the compact toroid injection experiment (CTIX) using a two-dimensional MHD code in an attempt to better understand the physics behind the compact toroids. The diagnostics contained within this code directly correlate with the actual diagnostics placed on the machine, which include interferometry measurements of the line-integrated electron density and magnetic field probes. Detailed comparisons between the experiments and simulations provide feedback required to further improve the simulations and also to provide information on parameters that are difficult to measure in experiments. These simulations have aided in the interpretation of experimental data on the acceleration dynamics of the compact toroid. They have also led to a better understanding of the mechanisms which limit the velocity to which these compact toroids can be accelerated in a given gun geometry.

Experiments have also been performed to address the issue of SCT fueling of high beta tokamak plasmas. There are several important issues to be investigated. These issues include a potential reconnection of the SCT before entering the tokamak due to the long-range toroidal field in comparison to the diamagnetic field. Another issue that must be investigated is the effect of hydrodynamic plasma pressure as beta is increased.

To study the interaction of an accelerated SCT with a tokamak equilibrium field structure, we have calculated the high beta equilibrium of a spherical tokamak. As the poloidal beta of the device becomes greater than one, the effect of the plasma is diamagnetic, causing the formation of a magnetic well inside the plasma region. As beta increases, the well depth increases accordingly. Experimentally, we have simulated the magnetic structure using vacuum field coils. The results of this experiment show that even with a short-range diamagnetic field in the plasma, the SCT penetration is improved.

\section{EFFECT OF THE EXTERNAL MAGNETIC FIELD INTERSECTING A DRIFT TUBE (N. Fukumoto)}

Compact Toroids (CT's) were injected along a drift tube into a perpendicular magnetic field, using a magnetized coaxial plasma gun (MCPG). We want to understand how a CT penetrates this magnetic field in CT particle fueling applications. We have observed several different phenomena. The CT is decelerated upon penetrating the field $B_{e x}$, and the penetration depth is shorter for higher fields. In our model of this deceleration, in which $v_{C T}$ is the velocity of the CT, there is a polarization electric field induced in the direction of $-v_{C T} \times B_{e x}$. This electric field is short-circuited through the external conducting drift tube, but a polarization current $\mathbf{J}_{\text {pol }}$ flows in the direction of $\mathbf{v}_{\mathrm{CT}} \times \mathbf{B}_{\mathrm{ex}}$ in the CT. As a result, the force $J_{p o l} \times B_{e x}$ decelerates the CT. We have observed the polarization current. 
A CT also shifts vertically. The shift motion depends on not only the direction of $B_{\text {ex }}$ but the polarity of the inner electrode of the MCPG. The direction of the CT shift is that of $J_{z} \times B_{e x}$, where $J_{z}$ is the gun current that flows from or to the inner electrode along the $z$-axis (the center axis of the inner electrode). A part of the current flows as a poloidal current of the CT. We showed three models of this shift motion.

\section{PARTICLE CONTROL EXPERIMENTS IN SSPX}

E.B. Hooper, H.S. McLean

The Sustained Spheromak Physics Experiment (SSPX) addresses the physics of spheromak formation, current buildup, sustainment and decay. Particle control (of impurities and fuel gas) is essential for good spheromak performance since current buildup is a sensitive function of plasma resistivity $\eta \sim Z_{\text {eff }} T_{e} e^{-1.5}$ The power balance between ohmic heating and radiative losses leads to a $T_{e}$ requirement for the minimum normalized current density, $\frac{j}{n}>10^{-14}$ A-m.

Spheromaks typically have close-fitting walls to provide MHD stability. This proximity can lead to enhanced plasma-surface interactions, for example, sputtering of hi-Z wall metals and desorption of loosely bound low Z impurity atoms. In SSPX, impurity control is obtained by tungsten spray coating of the walls, Ti gettering of the lower vessel, baking, glow-discharge cleaning, and attention to good vacuum practices. Impurity line radiation measurements in the VUV show $\mathrm{O}, \mathrm{C}$, and $\mathrm{N}$ as the major contributors. Emission goes up with increased bank energy, but is insensitive to the amount of gas injected or prefilled for plasma discharge. Bolometric measurements indicate a large fraction $(>50 \%)$ of input power is radiated away.

Density control in SSPX largely results from careful design of the flux conserver, magnetic field shaping, and the same surface conditioning used to reduce impurities. Open field lines intersecting the flux conserver are minimized by smoothly shaping the flux conserver to match magnetic fields produced by a main solenoid coil and two shaping coils. The flux conserver is 0.5 inch thick copper to reduce diffusion of field into the walls. Surface treatments (baking and GDC) reduce the wall gas inventory and the recycling coefficient.

At present, density during formation reaches as high as $2 \times 10^{21} \mathrm{~m}^{-3}$, which then falls rapidly to $\sim 2 \times 10^{20} \mathrm{~m}^{-3}$ several 10 s of microseconds after the injection current drops below the critical injection value and the spheromak detaches from the source. The density then stays relatively constant for $0.5 \mathrm{msec}$ and then decays linearly to zero during the next $0.5 \mathrm{msec}$. In the detached phase, the density and density decay rate is insensitive to initial gas puff pressure or initial prefill conditions. Normalized current density $\mathrm{j} / \mathrm{n}$ in the decay phase has been measured at $\sim 2 \times 10^{-15} \mathrm{~A}-\mathrm{m}$, confirming conditions are below that required for radiation burnthrough.

Initial results with titanium gettering of the flux conserver walls, accompanied by a change in the flux configuration, have shown a dramatic reduction in radiation levels and a three-fold increase in energy confinement time from 0.4 to 1.4 milliseconds. Carbon lines in the VUV disappear, indicating burn-through of carbon impurities, leaving oxygen as the main radiator. The lowered radiation levels last for one or two shots after gettering. 
